SUPPORTING INFORMATION APPENDIX

for

\title{
Distribution of Colloid Particles onto Interfaces in Partially Saturated Sand
}

Yuniati Zevi, ${ }^{1}$ Annette Dathe, ${ }^{1}$ John F. McCarthy, ${ }^{2}$ Brian K. Richards, ${ }^{1 *}$ Tammo S. Steenhuis ${ }^{1 * *}$

${ }^{1}$ Department of Biological \& Environmental Engineering, Cornell University, Ithaca NY 14853

${ }^{2}$ Department of Earth \& Planetary Sciences, University of Tennessee, Knoxville TN 37996-1410

\begin{tabular}{|l|l|}
\hline \multicolumn{2}{|l|}{ Table SI-1. Figures in this appendix } \\
\hline Figure & Description \\
\hline SI-1 & Bright field imaging system \\
\hline SI-2 & Detail of bright field imaging system (dimensions in $\mathrm{cm}$ ) \\
\hline SI-3 & Confocal laser infiltration chamber (plan view; dimensions in cm) \\
\hline SI-4 & Retention of slightly hydrophobic colloids at grain surface imperfections. \\
\hline
\end{tabular}

Table SI-2. Video clips viewable at the supporting documents website:

[***Editor - insert site link information here]

\begin{tabular}{|c|l|c|}
\hline \multicolumn{2}{|l|}{ Video number, experiment number and title } & $\begin{array}{c}\text { Related } \\
\text { to Figure }\end{array}$ \\
\hline 1 & A1: 3D confocal reconstruction of water films and meniscii on sand grains & 2 \\
\hline 2 & A2: dyed water movement along water films & 3 \\
\hline 3,4 & B1: Retention of hydrophilic colloids at $\mathrm{AW}_{\mathrm{m}} \mathrm{S}$ interface & 5 \\
\hline 5,6 & B2: Retention of mixed hydrophilic colloids at the $\mathrm{AW}_{\mathrm{m}} \mathrm{S}$ interface & 6 \\
\hline 7 & B3: Retention of slightly hydrophobic colloids on grain surface (WS interface) & $7 \mathrm{e}-\mathrm{f}$ \\
\hline 8 & B3: Retention of slightly hydrophobic colloids at the $\mathrm{AW}_{\mathrm{m}} \mathrm{S}$ and $\mathrm{AW}$ interfaces & $7 \mathrm{a}-\mathrm{d}$ \\
\hline 9 & $\begin{array}{l}\text { B3: Slightly hydrophobic colloids retained ingrain surface imperfections (WS } \\
\text { interface) }\end{array}$ & SI-4 \\
\hline
\end{tabular}




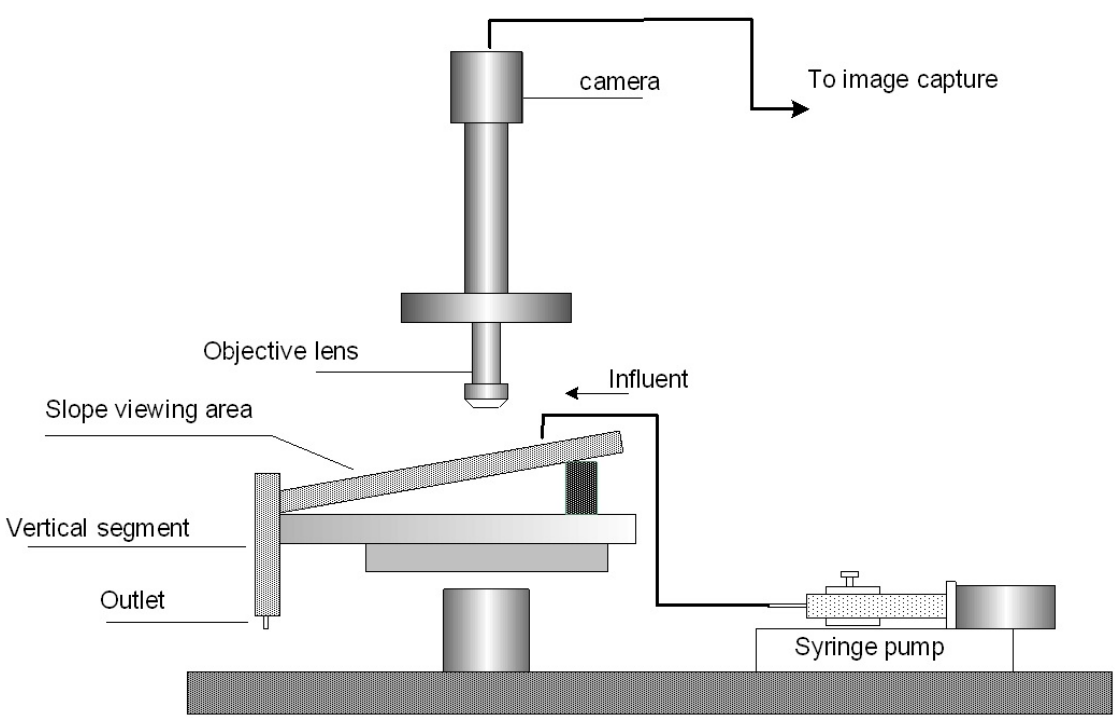

Figure SI-1. Bright field imaging system.

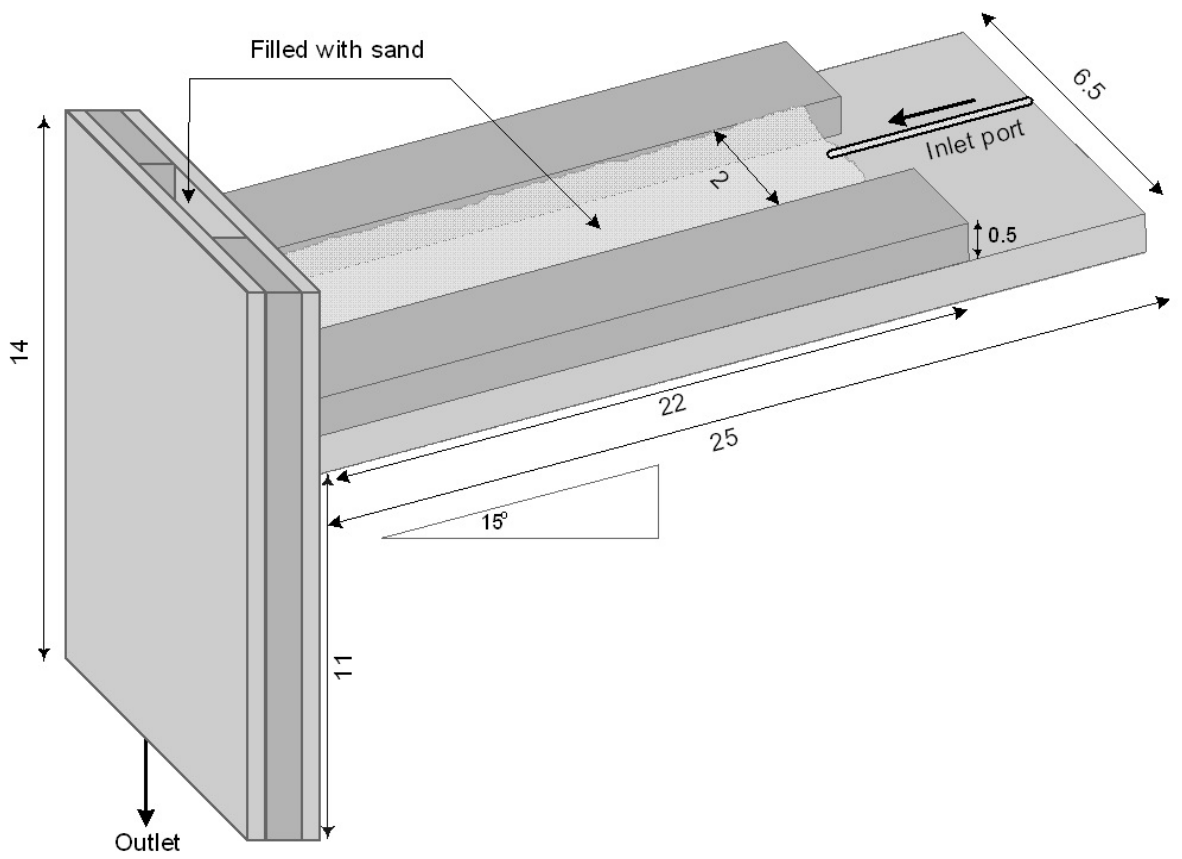

Figure SI-2. Detail of bright field infiltration chamber (dimensions in $\mathrm{cm}$ ). 


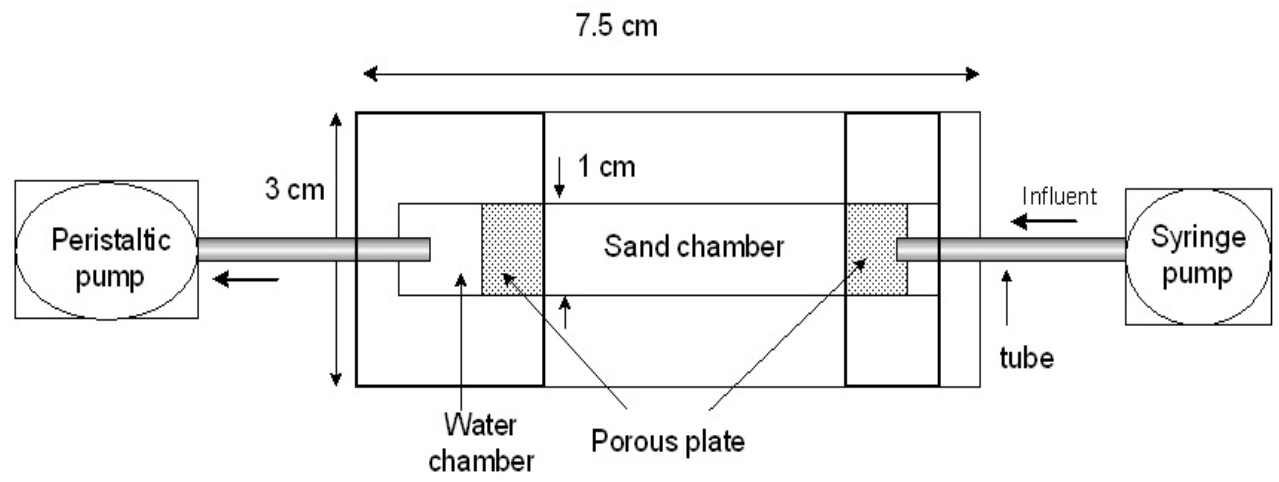

Figure SI-3. Confocal laser infiltration chamber (plan view; dimensions in $\mathrm{cm}$ ).
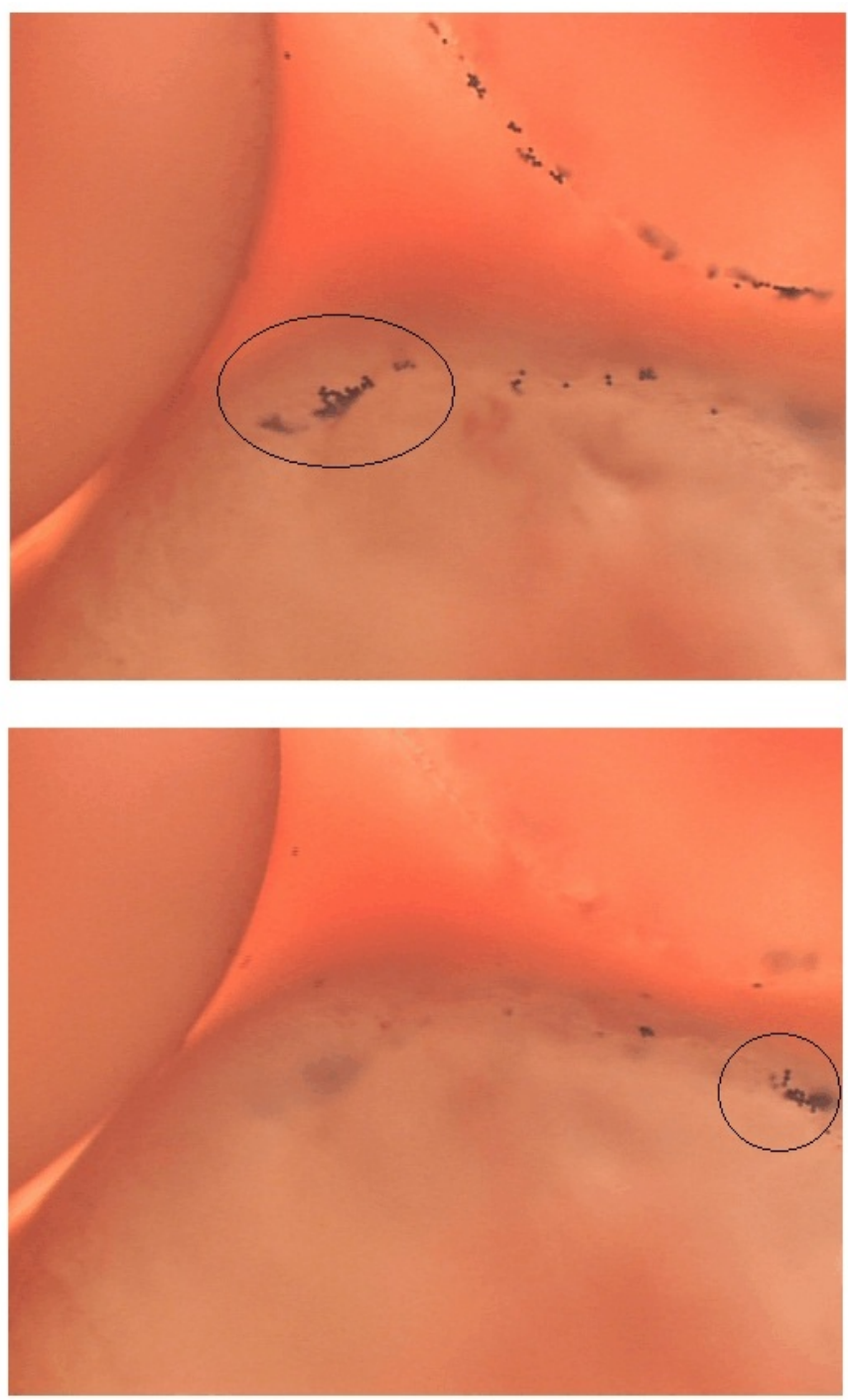

Figure SI-4. Retention of slightly hydrophobic colloids at grain surface imperfections (circled areas). 